Revue d'histoire de l'Amérique française

REVUE D.HISTOIRE DE L'AMÉRIQUE FRANÇAISE

\title{
La trahison de Bigot dans le roman historique canadien
}

\section{Maurice Lemire}

Volume 22, numéro 1, juin 1968

URI : https://id.erudit.org/iderudit/302753ar

DOI : https://doi.org/10.7202/302753ar

Aller au sommaire du numéro

Éditeur(s)

Institut d'histoire de l'Amérique française

ISSN

0035-2357 (imprimé)

1492-1383 (numérique)

Découvrir la revue

Citer cet article

Lemire, M. (1968). La trahison de Bigot dans le roman historique canadien. Revue d'histoire de l'Amérique française, 22(1), 65-88.

https://doi.org/10.7202/302753ar d'utilisation que vous pouvez consulter en ligne.

https://apropos.erudit.org/fr/usagers/politique-dutilisation/ 


\section{LA TRAHISON DE BIGOT}

\section{DANS \\ LE ROMAN HISTORIQUE CANADIEN *}

François Bigot occupe une place spéciale dans nos lettres. Le roman encore plus que l'histoire lui a buriné des traits de monstre. Dans une étude très savante ${ }^{1}$, M. Guy Frégault s'est attaché à lui restituer sa véritable figure. D'après ses conclusions, le dernier intendant ne serait pas un saint, loin de là, mais un fonctionnaire colonial dont la moralité ne tranche pas tellement sur celle de ses collègues. Lors du procès resté célèbre dans les annales sous le nom d'Affaire $d u$ Canada, la justice royale a cherché des boucs émissaires pour couvrir l'incurie du gouvernement pendant la guerre de Sept ans. Bigot fut la principale victime. Les Canadiens avaient aussi leurs raisons d'incriminer l'intendant; très tôt l'imagination populaire se mit de la partie. Quand en 1836, Amédée Papineau écrivit Caroline, il ne fit que transcrire une légende orale qui circulait dans le peuple depuis combien d'années ? nous n'en savons rien. Puis l'histoire de Bigot s'est toujours enrichie: en 1872, Marmette publiait L'Intendant Bigot, en 1877, William Kirby, Le Chien d'or, en 1886, Edmond Rousseau, Le Château de Beaumanoir, en 1910, Adèle Bibaud, Un épisode de la guerre de Conquête, et en 1926, Alexandre Huot, Le trésor de Bigot. Pourquoi Bigot a-t-il fait couler tant d'encre ? Pourquoi les romanciers en particulier l'ont-ils accaparé ainsi ? Voilà la question à laquelle nous allons essayer de répondre.

* Cette étude fait le chapitre II de la seconde partie d'une thèse de doctorat ès lettres présentée à l'Université Laval en septembre 1966 sous le titre général Les grands thèmes nationalistes du roman historique canadien-français.

${ }^{1}$ Guy Frégault, François Bigot, Administrateur français (2 vol., Les Etudes de l'Institut d'histoire de l'Amérique française, 1948). 
La vie scandaleuse de l'intendant ne manque pas d'intérêt; elle fournit un canevas propice aux intrigues les plus compliquées et un romancier de talent comme Kirby a montré le parti qu'on pouvait en tirer. Mais prétendre que les écrivains canadiens se sont laissés attirer par l'attrait du scandale serait faire fausse route. Leurs principes étaient trop rigides pour qu'on les soupçonne un seul instant d'un tel dessein. Toutefois, les manigances de Bigot avaient tout le piquant nécessaire pour assaisonner une leçon, même sévère, de patriotisme. Mais quelle leçon tirer de pareilles turpitudes?

Le gouvernement de Bigot couvre les pages les plus sombres de notre histoire, celles qui ont scellé la destinée du peuple pour des siècles. Il importait au plus haut point pour notre honneur national d'interpréter favorablement les événements qui entourent la conquête. C'était bien assez de subir les conséquences de la défaite sans en essuyer la honte. Bigot de nouveau fut le bouc émissaire, celui qu'on allait charger de tous les torts, de toutes les erreurs.

Dans les romans consacrés à l'intendant Bigot, nous remarquons deux mouvements très distincts: d'un côté, un essai de valorisation des Canadiens, et de l'autre, une charge contre Bigot et l'administration métropolitaine. On tente de dégager la responsabilité des nôtres en rejetant tout l'odieux sur les administrateurs français. On veut éviter de laisser croire les Canadiens vaincus faute de courage et de bravoure. Peu importe que tout soit perdu pourvu que l'honneur soit sauf.

Deux romans donnent de l'administration Bigot une interprétation voisine. L'Intendant Bigot de Marmette et le Château de Beaumanoir de Rousseau. L'intrigue des deux œuvres se ressemble au point de laisser croire Rousseau largement tributaire de son aîné. Dans les deux récits, Bigot s'énamoure d'une jeune fille noble mais pauvre. Il la fait enlever par ses sbires et séquestrer à son château de Beaumanoir. Pendant sa captivité, la jeune fille surprend la conspiration de Bigot avec des émissaires anglais. Elle réussit à s'évader et livre le secret à Vaudreuil. Mais il est trop tard, les Anglais occupent déjà les Plaines 
d'Abraham. Berthe de Rochebrune, l'héroïne de Marmette, et Claire de Godefroy, celle de Rousseau, sont toutes les deux amoureuses de jeunes officiers canadiens intimement liés au gouverneur de Vaudreuil. Louis Gravel est secrétaire du gouverneur, Raoul de Beaulac, un confident. Ces jeunes gens représentent en quelque sorte le parti du peuple contre les affidés de Bigot. La jeune fille qu'ils cherchent à délivrer pourrait bien personnifier la nation prisonnière des agents de la Friponne.

Tout au long de son récit, Joseph Marmette décrit les prodiges d'héroïsme du peuple pris entre deux feux, les Anglais d'un côté, et les complices de Bigot de l'autre. Dès les premières pages, il met dans la bouche du vieux soldat Rochebrune ces paroles prophétiques qui résument son interprétation des événements de la Conquête:

Ecoutez ! continua le vieillard, comme saisi d'une subite inspiration. L'ennemi s'avance ... j'entends au loin le bruit de son avant-garde qui franchit la frontière... Manquant de vivres et de munitions, nos soldats, inférieurs en nombre, retraitent pour la première fois ... l'Anglais les suit... il s'approche ... il arrive... et je vois ses bataillons entourer nos murailles... Bien qu'épuisés par la disette et la lutte, le soldat, le milicien, le paysan disputent avec acharnement à l'étranger le sol de la patrie... La victoire va peut-être couronner leur courage... Mais non! des hommes éhontés se sont dit: "Le moment est venu d'éteindre le bruit causé par nos exactions sous le fracas de la chute du pays que nous avons si mal administré... Entendons-nous avec l'Anglais ..." et guidés par un traître, je vois nos ennemis tant de fois vaincus, surprendre et écraser nos frères ! ${ }^{2}$

Ainsi, sans la trahison de Bigot, nos soldats épuisés par la disette et la lutte auraient pu repousser encore l'envahisseur.

En effet, les Canadiens n'ont rien épargné pour le salut du pays. Ils laissent réduire leurs fermes en cendres et ravager

2 Joseph Marmette, L'Intendant Bigot (Montréal, 1872), 9.

3 Ibid., 39. 
tout le pays des alentours. Ils "avaient fait d'avance le sacrifice de tout ce qui leur était cher" 3 . Affamés par les malversations de Bigot, ils se demandent s'ils pourront tenir longtemps: "Et tu crois, dit Raoul de Beaulac, que lorsque nous serons sans asile, sans munitions, sans pain et sans argent, nous pourrons tenir longtemps tête à un ennemi bien muni de tout ce qui nous manque ? Non, Lavigueur, aux yeux de tous les gens éclairés, notre situation est désespérée... " Et malgré la certitude de ne rien retirer de leur sacrifice, ils n'hésitent pas à donner leur vie.

Cette générosité, d'après Marmette, est digne des plus grands éloges :

N'était-ce pas de l'héroïsme que l'acte de ces gentilhommes et de ces paysans qui couraient à la mort, les uns persuadés qu'elle serait inutile au salut du pays, et les autres confiants dans le succès de leurs armes et comptant toujours sur des secours que la France ne leur envoyait plus depuis longtemps $?^{5}$

La défaite qu'on leur impute comme une honte, loin de les déshonorer, les grandit. Elle nous porte aujourd'hui à rougir d'eux parce que nous n'avons plus la noblesse de comprendre leur désintéressement: "Nous sommes d'autant plus émerveillés aujourd'hui de la lutte acharnée qui retarda la conquête, qu'énervés par de longues années de paix, et le cœur racorni par cette fièvre des intérêts matériels qui va courant par le monde et ronge tous les peuples, nous ne savons plus agir que pour des motifs froidement calculés et pesés au poids d'un bien-être assuré 5." La défaite n'a done rien de déshonorant pour nos ancêtres; ils ont fait plus que leur devoir. Les blâmes doivent retomber sur d'autres.

Bigot est le grand responsable de la perte de la colonie. Voyant venir l'heure de la reddition des comptes, il voulait sauver du désastre la fortune colossale ${ }^{6}$ amassée aux dépens des Cana-

4 Ibid., 41.

5 Ibid., 41.

6 Après Marmette, Alexandre Huot a repris cette légende des richesses fabuleuses de Bigot dans un roman intitulé: Le trésor de Bigot (Montréal, E. Garand, 1926). 
diens. La découverte de ses malversations pouvait tout compromettre. Mieux valait trahir que de tout perdre. Il fait le raisonnement suivant à son complice Vergor :

Bon ! dites-moi, maintenant, mon cher Vergor, ne vous semble-t-il pas que si le pays passait immédiatement entre les mains des Anglais, il nous serait assez aisé de cacher une grande partie de nos méfaits sous les ruines de cette colonie ? Ne croyez-vous pas qu'il serait bien difficile à messieurs nos juges, si toutefois il nous faut comparaître devant un tribunal, de nous forcer à un compte rendu très exact de notre administration ? La belle occasion pour rejeter presque toutes les dépenses sur les frais de guerre ! 7

L'intendant noue alors des intelligences avec l'ennemi. Son plan est très simple. Montcalm attend l'ennemi du côté de la rivière Saint-Charles; il y a cantonné le gros de son armée. Impossible aux Anglais d'attaquer de ce côté. Impossible non plus d'attaquer de face; les flancs du cap sont trop escarpés. Reste l'Anse-auFoulon. Là encore, débarquement difficile à cause de la falaise. Mais en s'assurant la complicité des gardiens, l'accès devient possible sans donner l'alerte. Donc pour parvenir aux Plaines d'Abraham et prendre Québec à revers, il suffit de la complicité de la vigie de l'Anse-au-Foulon. Or cet homme, de Vergor, est un factotum de l'intendant. Bigot l'a fait nommer à ce poste dernièrement dans un dessein bien précis :

Bigot: Pouvez-vous me dire à qui vous devez le commandement de ce poste important du Foulon, que l'on vous a confié depuis quelques jours?

Vergor: Je n'en sais rien.

Bigot: Je le crois bien ; car on n'a plus grand' confiance (sic) en vous depuis la capitulation de Beauséjour. Et il m'a fallu mettre bien des influences en mouvement pour vous faire nommer à ce poste de confiance. Je ne voulais pas me compromettre en le deman-

7 J. Marmette, L'Intendant Bigot, 55. 
dant moi-même pour vous. Vous comprendrez pourquoi quand je vous dis qu'il entre dans mon plan que vous... n'empêchiez pas trop les Anglais de forcer le passage du Foulon aux Plaines d'Abraham.

Vergor: C'est à dire que ... qu'il me faudra ... les laisser faire!

Bigot: Oui. ${ }^{8}$

Voilà comment Bigot livre la Nouvelle-France pour sauver sa fortune.

Marmette ne veut pas que l'on prenne cette interprétation pour de l'imagination. Il prétend lui donner des fondements historiques. Dans une longue note, il cite les documents qui justifient son hypothèse:

Tout en faisant la part du drame, je tiens à montrer que cette hypothèse de trahison est assez bien fondée. Aussi vais-je citer tout le passage des mémoires sur les affaires du Canada (p. 164, édition de 1838) qui à trait à la surprise du Foulon, en ayant soin d'en souligner les phrases qui viennent à l'appui de ma thèse.

"M. Wolfe, qui avait renforcé le camp de la Pointe-Lévy, semblait flatter les idées des Français: l'Amiral Saunders faisait aussi exécuter des manœuvres qui annonçaient une prochaine retraite. $\mathrm{Au}$ milieu de toutes ces espérances, on confia au Sieur de Vergor le poste du Cap-Rouge, au-dessus de Québec; on ne pouvait mieux seconder les intentions $d u$ général anglais, dont le but était de faire une descente sans être obligé d'attaquer l'armée retranchée. On avait consigné à cet officier de laisser passer des bateaux chargés de vivres qui devaient entrer dans Québec en se coulant le long du cap. Ce capitaine avait avec lui beaucoup d'habitants de Lorette dont le lieu était à la portée de ce poste; ils lui demandèrent permission d'aller aussi travailler pour lui sur une terre qu'il avait dans cette paroisse. $M$. Wolfe, averti à temps de la mauvaise garde de ce poste et du commandant à qui il avait affaire, disposa ses trou-

8 Ibid., 55. 
pes. Le Sieur de Vergor était dans dans la plus grande sécurité. On vint l'avertir qu'on apercevait des berges remplies de monde qui venaient sans bruit au-dessus et au-dessous de son poste. Il répondit que c'était des bateaux de munitionnaires et qu'on les laissât tranquilles." 9

Marmette croit ce texte suffisant pour accuser Bigot de trahison. Dans la conjoncture, il ne voit pas simplement des hasards mais des intentions. En nommant Vergor, on favorisait les desseins de Wolfe. Le romancier interprète: une personne intéressée a fait nommer Vergor; en l'occurrence il ne peut s'agir que de Bigot. Qui d'autre pourrait avoir intérêt à perdre la colonie ? "On avait consigné à cet officier de laisser passer les bateaux chargés de vivres qui devaient entrer dans Québec." N'est-ce pas là un euphémisme qui dit bien davantage ? Enfin, Vergor avait accordé congé à ses hommes. C'est une conséquence normale des ordres reçus. Le poste avait volontairement été démuni à l'approche de l'ennemi.

Pour Marmette, la trahison de Bigot n'est donc pas une légende mais un fait historiquement prouvé. C'est pourquoi il est faux de considérer les Canadiens comme des vaincus; ils ont été trahis. Ceci importe souverainement à leur honneur.

Edmond Rousseau poursuit les mêmes buts que Marmette: excuser les Canadiens de la défaite de 1760 . Cependant il écrit à une époque où les esprits sont échauffés par la révolte de Riel. Par suite de l'agitation des Métis du Manitoba, le Gouvernement fédéral a recruté un contingent de volontaires pour mâter les rebelles. Les Canadiens français n'ont pas montré autant d'enthousiasme que leurs compatriotes anglo-canadiens dans cette entreprise; la presse anglaise les a donc taxés de lâcheté. En réponse à ces accusations, Rousseau écrit: "Une certaine presse francophobe naguère encore, n'a pas craint de nous accuser de "lâcheté", de "cruauté", de "pillage". Il suffit pour venger notre honneur national outragé et faire repentir nos détracteurs, de mettre en regard les actions de nos pères et celles de leurs

9 Ibid., 58. 
aïeux ${ }^{10}$." Sous forme de roman, il compare donc le comportement des partis adverses lors de la conquête.

Les Canadiens n'ont qu'un tort, avoir été vaincus. Mais leur honneur n'est pas en cause: ils ont succombé sous le poids de l'adversité, desservis par les circonstances et les hommes.

Toute la responsabilité de la défaite repose sur Bigot et par ricochet sur la cour de France. Rousseau présente l'intendant comme un favori de la Pompadour qui doit à la courtisane royale sa magistrature à Louisbourg et à Québec. Nommer un tel administrateur après les preuves d'incompétence qu'il avait données, prouve l'indifférence de la cour à l'égard de la colonie. En effet, Louis XV, sous l'influence de sa maîtresse, en est venu à abandonner presque complètement ses territoires d'outre-mer: "La colonie, malgré le courage de ses habitants, en dépit de l'héroïsme de nos troupes et du talent de nos généraux, n'en est pas moins à deux doigts de sa perte, grâce (sic) au manque de cœur d'un monarque qu'on a l'audace d'appeler le bien-aimé, et qui n'est que le jouet d'un cotillon comme la Pompadour ${ }^{11}$." Pas surprenant que l'on ferme les yeux sur les agissements de Bigot.

Fort de la complicité de la cour, l'intendant, avec un sens merveilleux de l'organisation, met sur pied de grandes entreprises commerciales comme la Friponne et la Grande Compagnie. Il profite de la pauvreté d'honnêtes gens pour les compromettre dans ses affaires frauduleuses. Il donne gracieusement à M. de Godefroy, le père de l'héroïne, un nombre imposant de parts de sa compagnie pour le tenir à sa merci et l'utiliser au moment voulu. Ainsi l'intendant et ses comparses peuvent détourner toutes les ressources de la colonie à leur profit: tandis que la Nouvelle-France est près de succomber sous le poids du malheur et de l'abandon, tandis que partout règne la disette, Bigot, Cadet, Péan, Varin et un grand nombre d'autres s'enrichissent en volant les secours du roi et en pressurant le peuple ${ }^{12}$.

10 Edmond Rousseau, Le Château de Beaumanoir (Lévis, 1886), VI.

11 Ibid., 5.

12 Ibid., 131. 
La misère consécutive à un tel pillage est incroyable. Rousseau en donne une idée en citant un extrait d'une lettre de M. de Doreil à M. de Vaudreuil :

Le peuple périt de misère; les Acadiens réfugiés ne mangent depuis quatre mois que du cheval et de la morue sans pain; il en est déjà mort plus de trois cents. Le peuple canadien en est toujours réduit, ainsi que nous, au quart de livre de pain par jour. A l'égard de la viande, on oblige ceux qui sont en état d'en manger, de prendre moitié cheval à six sous la livre. Nos soldats sont à la demi-livre de pain par jour depuis le premier novembre, trois livres de bœuf, deux livres de pois et deux livres de morue par huit jours. Ils prennent leur mal en patience ${ }^{13}$.

Bigot réussit d'autant mieux ses filouteries qu'il exploite le sentiment patriotique des Canadiens. Toute cette misère, toutes ces privations, le peuple les "supporte avec patience, avec héroïsme parce qu'il les croit nécessaires pour sauver le pays" ${ }^{14}$.

Normalement la disette, la mauvaise administration, les injustices auraient dû refroidir le zèle des coloniaux pour le service du roi. Au contraire, réduits à leurs seules ressources déjà bien entamées par la voracité des concussionnaires, les Canadiens espèrent conserver la colonie par leurs propres moyens. Lors du siège de Québec, on voit bien jusqu'où va leur héroïsme:

Les habitants de la colonie montrèrent un héroïsme qui fait la gloire immortelle de nos aïeux. Que l'on nous permette de donner le jugement qu'en portait un des officiers généraux de l'époque: “On ne comptait, dit-il, sur une armée aussi forte parce que l'on ne s'était pas attendu à voir un si grand nombre de Canadiens; on n'avait eu l'intention d'assembler que les hommes en état de soutenir les fatigues de la guerre; mais il régnait parmi ce peuple une telle émulation que l'on vit arriver au camp des vieillards de quatre-vingts ans et des enfants de douze à treize ans qui ne voulurent jamais profiter de l'exemption accordée à leur âge. Jamais sujets ne furent plus

13 Ibid., 158.

14 Ibid., 159. 
dignes de la bonté de leur souverain, soit par la constance de leur travail, soit par leur patience dans les peines et les misères qui, dans ce pays, ont été extrêmes." 15

Ainsi, les déprédations de Bigot et l'abandon de la cour n'ont pas eu raison du patriotisme des colons. Sans la trahison de Bigot, les Canadiens n'auraient pas connu la défaite.

Dans le roman de Rousseau, Bigot sent aussi que la mesure est pleine; M. de Vaudreuil, au courant de ses malversations, s'apprête à le dénoncer au roi. "Donc, se dit Bigot, il faut que la colonie périsse pour nous assurer l'impunité ${ }^{16}$." Dans la nuit, il reçoit un émissaire anglais à Beaumanoir pour régler les conditions de la trahison. L'intendant convient de cinq cent mille livres pour lui et de trois cent mille pour chacun de ses complices. En retour il livre le passage de l'Anse-au-Foulon avec la complicité de Vergor. A partir de ce moment-là, l'héroïsme le plus extraordinaire ne servirait plus de rien. En vrais soldats, les nôtres combattent uniquement pour l'honneur.

Après la débandade des Plaines d'Abraham, rappelle Rousseau, les Canadiens furent les seuls à offrir une résistance véritable. Profitant des moindres bosquets, ils disputent chèrement chaque pied de terrain à l'ennemi. La fortune si mesquine à leur endroit leur réserve cependant une éclatante revanche; le romancier raconte en détail la bataille de Sainte-Foye ${ }^{17}$. Après une victoire remportée dans des conditions aussi difficiles, les nôtres ne doivent plus avoir honte de rendre les armes; ils ont vraiment droit à l'éloge que Vaudreuil fait d'eux devant la cour de Paris :

En général, les Canadiens semblent nés soldats; une éducation mâle et toute militaire les endurcit de bonne heure à la fatigue et au danger. Le détail de leurs expéditions, de leurs voyages, de leurs entreprises, de leurs négociations avec les naturels du pays offre des miracles de courage, d'activité, de

15 Ibid., 165.

16 Ibid., 207.

17 Le Château de Beaumanoir, 264-268. 
patience dans la disette, de sang-froid dans les périls, de docilité aux ordres des généraux qui ont coûté la vie à plusieurs sans jamais ralentir le zèle des autres. Ces commandants intrépides avec une poignée de Canadiens et quelques guerriers sauvages, ont souvent déconcerté les projets, ruiné les préparatifs, ravagé les provinces et battu les troupes anglaises, huit à dix fois plus nombreuses que leurs détachements (18).

Une conduite aussi héroïque n'a pas à nous faire rougir. Au contraire ce sont les Anglais qui devraient avoir honte du comportement de leurs troupes pendant la campagne de 1759 .

Le romancier rappelle l'attaque de l'armée anglaise contre une population sans défense, composée uniquement de femmes, d'enfants et de vieillards. Sans raison les ennemis ont incendié les fermes, les villages, ravagé les moissons et capturé les bestiaux. Ils livraient ainsi toute la population du Bas-du-Fleuve à la famine, à la veille même de l'hiver. Au chapitre XXXVIII, Rousseau abandonne le ton de l'objectivité pour retourner contre les Anglais les accusations de lâcheté, de brigandage et de pillage proférées par les journalistes ontariens. Il cite à l'appréciation du lecteur de longs extraits d'une lettre de l'évêque de Québec à Louis XV en date du 9 novembre 1759. Le prélat y décrit la misère des habitants des villes et des campagnes par suite de la guerre. Les conquérants ont ruiné le pays. Rousseau, conscient de l'arbitraire de tels procédés, croit de bonne guerre de se placer sur le même pied que les détracteurs ontariens :

Nous ne sommes pas plus anglophobes qu'un autre; mais dans un temps où une certaine presse anglaise ne se gêne pas pour accuser, sans le moindre prétexte, les Canadiens français de lâcheté, de barbarie, nous avons bien le droit de rappeler ce que furent leurs pères, avec quelle sorte d'humanité les aïeux de nos détracteurs d'aujourd'hui traitèrent les nôtres à cette époque ${ }^{19}$.

18 E. Rousseau, Le Château de Beaumanoir, 274.

19 Ibid., 169. 
Ainsi l'honneur des Anglais est plus compromis que le nôtre dans la campagne de 1759 .

Dans le roman Avant la conquête, un épisode de la guerre de 1757, Adèle Bibaud essaie, elle aussi, de disculper les vaincus de 1759 en reportant la responsabilité de la défaite sur Bigot et sur la cour de France.

Louis XV et sa cour corrompue sont les grands responsables de la perte du Canada. Au milieu des bals et des fêtes, le roi oublie ses braves sujets de la Nouvelle-France. Pas un courtisan n'a le cœur d'intercéder pour eux:

Pourquoi le roi n'envoie-t-il pas de nouvelles recrues afin de tenir nos ennemis en respect ? Que son inertie est coupable, tandis qu'ici des centaines de braves soldats se battent et versent leur sang pour la patrie, on les oublie, là-bas, on ne songe qu'aux plaisirs et qu'aux fêtes. Ah ! si notre souverain pouvait voir couler les larmes des mères, des épouses et des sœurs qui comptent parmi les morts leurs affections les plus chères, peut-être se sentirait-il ému et n'assumerait-il pas plus longtemps une telle responsabilité devant Dieu. Ces courtisans qui l'entourent ne se fatigueront-ils jamais de ce faste, de ces pompes, de ces fêtes. Au lieu de ces flatteries incessantes qu'ils prodiguent au monarque, ne pourraient-ils pas glisser une parole en faveur de ces héroïques Canadiens, de ces valeureux soldats qui donnent leur vie pour conserver à la France un pays auquel son roi ne semble plus tenir ${ }^{20}$.

Montcalm et ses lieutenants déploient tous leurs talents pour palier les négligences de la France mais ils n'obtiennent pas les succès qu'ils méritent à cause de la corruption de l'administration: Bigot et ses amis approvisionnent mal l'armée. Montcalm s'en plaint à M. de Marville: "Je déplore que le transport des vivres et des munitions soit, pour la plupart des employés corrompus et prévaricateurs du gouvernement, un moyen de s'enrichir. On ne craint pas de piller l'argent et les effets du roi ${ }^{21}$."

${ }^{20}$ A. Bibeau, Avant la conquête (Montréal, The Montreal Printing and Publishing Co., 1904), 43.

21 Ibid., 60. 
A mesure que l'armée se couvre de gloire à Monongahéla, à Chouaguen, à Carillon, elle épuise les maigres ressources de la colonie. Chaque campagne est l'occasion de spéculations et de concussions nouvelles. Bigot profite de la précarité de la situation pour multiplier les comptes; Montcalm s'en indigne:

Tous ces comptes ont été payés ? demande-t-il à Bourlamaque. Bourlamaque: Oui, général, ordre de l'intendant Bigot. Montcalm: Cependant, un grand nombre étaient faux et demandés pour des articles qui n'ont jamais été fournis à l'armée. C'est ainsi que tandis que des milliers de braves guerriers s'efforcent de soutenir, en ce pays, la gloire militaire, des administrateurs infidèles prennent à tâche de dilapider les finances. Ne sommes-nous pas dans un temps assez critique ? faudra-t-il encore être contraints de réduire la ration des troupes pour enrichir d'indignes fonctionnaires ? ${ }^{22}$

Ainsi desservis, les braves de Montcalm peuvent-ils encore espérer la victoire finale ? Ils sont infailliblement voués à la défaite; à leur nom, les historiens accoleront pour toujours l'épithète de vaincus: "Que diront les temps futurs si Montcalm est vaincu, si l'Angleterre est victorieuse ? Il faut tripler les hommes, les faire sortir de terre, il faut lutter contre toute espérance. Comprenez-vous, Robert, la position du général en chef de l'armée d'Amérique ? Ne voyez-vous pas, comme moi, son nom inscrit par l'implacable écrivain au nombre de ceux chez qui la valeur fait défaut, incapable de commandement ? ${ }^{23}$ " Voilà la grande souffrance, passer pour des incapables au même titre que tous les vaincus quand les victoires se sont succédé sans interruption tant que les approvisionnements ont été suffisants. En fait ce serait une injustice de honnir les troupes françaises: une seule défaite ne peut ternir tout un passé de gloire:

Général, vous oubliez que la mémoire du vainqueur de Carillon ne peut être ternie. Louis XIV fut défait; mais il n'en est pas moins resté Louis le Grand, et la postérité redira de siècle en siècle ses exploits comme elle reconnaîtra la bravoure du général Mont- 
calm et si, malgré les plus nobles efforts nous succombons, la mère patrie ne pourra jamais regretter de vous avoir choisi, car qui mieux que vous, général, aurait pu maintenir le drapeau français en ces contrées que le roi abandonne, comme une charge inutile, une dépense onéreuse, pour lutter, sans forces, avec un ennemi dix fois supérieur en nombre ${ }^{23}$.

Les Canadiens auraient donc mauvaise grâce de rougir de leur passé quand par ailleurs leurs ancêtres, inférieurs en nombre et en équipement, ont porté victorieusement la guerre sur tous les points du continent et tenu tête à l'ennemi jusqu'à l'épuisement, et cela malgré les déprédations continuelles des concussionnaires.

Adèle Bibaud n'accuse pas Bigot de trahison. Certes, l'intendant a fortement contribué à la ruine de la colonie par ses malversations, mais rien n'indique qu'il ait été d'intelligence avec l'ennemi. Toutefois, elle rapporte, elle aussi, le lâche comportement de Vergor à l'Anse-au-Foulon: "M. de Vergor qui commandait en cet endroit devait défendre le passage de la côte, se laissa surprendre quoique la nuit ne fut pas obscure, par un détachement de cinquante hommes; et cet indolent commandant qui, trois ans auparavant, s'était si mal défendu dans son fort de Beauséjour, se laissa déloger sans opposer une grande résistance ${ }^{24}$." Plus loin, la romancière affirme que le commandant aurait pu "facilement empêcher ce débarquement". Rien n'indique qu'il y ait eu connivence avec Bigot.

Bien qu'Adèle Bibaud n'accuse pas formellement Bigot de trahison, elle ne l'en tient pas moins responsable de la défaite. Elle n'ajoute pas d'idées nouvelles à ce que Marmette et Rousseau ont déjà affirmé; elle fait cependant chorus.

Deux romans canadiens-anglais donnent une version assez identique de la conquête, Le Manoir de Villerai de Mme Eleonore Leprohon et Le Chien d'or de William Kirby. Le premier raconte une idylle qui n'a que des liens très lâches avec la guerre de la conquête. La romancière note les événements historiques au fur et à mesure de son récit sans que les amours de M. de Montarville

24 Ibid., 158. 
et de Mlle de Villerai en soient affectées. Toutefois le cadre historique que Mme Leprohon donne à son roman reproduit le double mouvement que nous avons trouvé dans les romans canadiens-français, à quelques variantes près.

La responsabilité de la perte du Canada revient d'abord à la cour de Louis XV. Les divertissements royaux accaparent tous les fonds du trésor; il ne reste plus rien pour la colonie:

Cependant la colonie attendait avec impatience et anxiété les secours si instamment demandés à la mère patrie; mais celle-ci ou plutôt ses ministres, étaient plus soucieux de fournir l'argent nécessaire à la honteuse prodigalité de la cour scandaleuse de Louis XV et de ses royales favorites que de protéger leurs soldats et leurs colons qui combattaient si noblement pour l'indépendance d'une terre lointaine. La déclaration ridicule de madame de Pompadour, que le Canada, pays de déserts glacés et d'impénétrables forêts, avait déjà coûté plus qu'il ne valait, était suffisante pour engager un ministre esclave à abandonner ce malheureux pays à son triste sort ${ }^{25}$.

Malgré la vaillance des défenseurs de la Nouvelle-France, la France corrompue du XVIIIe siècle ne pouvait pas conserver sa colonie d'Amérique: elle consacrait toutes ses ressources aux plaisirs.

Au Canada, la série de victoires qui signalent les débuts de la guerre de Sept ans ne fut interrompue que par l'insuffisance des approvisionnements: la colonie se saignait à blanc pour défendre son territoire sans jamais refaire ses forces: "La situation du Canada, malgré les succès qui accompagnaient généralement ses armes devenait chaque jour de plus en plus triste et désespérée ${ }^{26}$." Le honteux péculat qu'exerçait Bigot mettait Montcalm dans une position d'infériorité compensée un temps seulement par la bravoure de ses soldats: "A cause de l'extrême cherté des provisions, et des reproches qu'on ne cessait de faire au gouvernement, reproches qui devenaient encore plus vifs par 74.

25 E. Leprohon, Le Manoir de Villerai (Montréal, Beauchemin, 1901), 26 Ibid., 84. 
le honteux système de pillage et de péculat exercé sous l'autorité de l'intendant Bigot, de Montcalm ne peut entrer en campagne pour suivre les mouvements des troupes anglaises que très tard l'année suivante ${ }^{28}$."

Mme Leprohon n'exclut pas la possibilité d'une trahison. Toutefois, elle n'accuse pas Bigot. Si Wolfe ne connaît pas le même sort que Walker dans le golfe Saint-Laurent, il le doit à la trahison d'un Canadien:

Ils (les Anglais) remontèrent le Saint-Laurent et arrivèrent à l'île d'Orléans le 25 juin, sans le moindre accident, malgré les nombreux périls et les difficultés multiples qui accompagnaient alors la navigation du fleuve. Cette bonne fortune était due en partie à la trahison du commandant d'une frégate française, Denis de Vitré, fait prisonnier par les Anglais pendant la guerre, et qui les avait conduits en sûreté à Québec, lieu de sa naissance. Il fut récompensé de cet acte infâme par une commission dans l'armée anglaise ${ }^{27}$.

Le nombre n'a donc pas suffi à vaincre les Canadiens, il a fallu la trahison. Toutefois la trahison de Vitré n'a pas la même conséquence que celle de Bigot. En livrant le passage de l'Anse-auFoulon, l'intendant livrait aussi la colonie: l'attaque surprise de Wolfe sur les plaines déjouait tous les plans de Montcalm. Tandis que les renseignements de Vitré privaient la colonie de ses défenses naturelles mais ne changeaient rien aux plans militaires. La rtahison de Vitré ne peut donc pas donner lieu à la même interprétation que celle de Bigot.

Le Chien d'Or de William Kirby est peut-être le roman le plus révélateur de cette période. Déjà en 1916, Pamphile Lemay écrivait: "Le Chien d'or a contribué, autant peut-être que l'œuvre de Garneau à nous rendre à nous-mêmes, par le récit et la description de la vie d'autrefois ${ }^{28}$." Ironie du sort, c'est un Canadien anglais qui devait exploiter notre patrimoine avec le plus de bonheur. En agençant les légendes du Chien d'or, de la Corriveau

27 Ibid., 305.

28 P. Lemay, Préface du Chien d'or (édition 1916), 10. 
et de Caroline telle qu'Amédée Papineau l'avait consignée, Kirby a réussi une fresque historique du Canada à la veille de la Conquête qui ne manque pas de saveur.

Bien que l'auteur soit très sympathique aux Canadiens français, nous ne pouvons cependant pas considérer les sentiments et les opinions qu'il prête à ses personnages comme l'expression du nationalisme canadien-français. Ce roman concerne cependant notre étude à un autre titre: il constitue une des rares expressions du nationalisme pancanadien au XIXe siècle.

Par l'interprétation qu'il donne de la fin du régime français, William Kirby poursuit visiblement les mêmes buts que les romanciers canadiens-français. Il désire réhabiliter les vaincus de 1760 en reportant tout le blâme de la défaite sur Bigot et sur la France. De plus, il espère qu'une fois rendus à eux-mêmes, une fois guéris de leur complexe de vaincus, les Canadiens français pourront s'associer à part égale à leurs compatriotes de langue anglaise pour former un grand Canada.

Kirby met d'abord l'accent sur les malversations de Bigot. L'intendant et ses associés de la Friponne cherchent à drainer toutes les ressources de la colonie dans leurs coffres; ils monopolisent le commerce intérieur, contingentent les importations, contrôlent la navigation sans autre opposition que celle du marchand Philibert. M. de la Galissonière, le gouverneur de l'époque, connaît les agissements de l'intendant mais il reste impuissant à les dénoncer; Bigot jouit d'un plus grand crédit à la cour. Créature de la Pompadour, cet administrateur profite de sa faveur depuis longtemps; n'a-t-il pas réussi, avant de venir au Canada, à déposséder à son avantage le comte Philibert de ses terres ? Aussi longtemps que la favorite le couvrira, il pourra impunément pressurer la colonie.

Pour stimuler encore davantage ses affaires, l'intendant recherche la guerre. La paix de 1748 serait une catastrophe pour les associés de la Grande Compagnie sans l'influence de Bigot et les ressources illimitées de son esprit. Au chevalier des Méloises qui s'inquiète, il répond: "Je me propose de faire tourner les 
événements à mon avantage." Il sait à qui s'adresser: les dames de Versailles "ce sont elles qui décident de tout" ${ }^{29}$. Il peut rassurer le chevalier en ces termes: "Une année ou deux de repos ne seraient point de trop peut-être pour ravitailler la colonie, et, alors, nous seront prêts encore à crocheter les serrures du temple de Bellone, et à crier avec plus de plaisir que jamais: "Vive la guerre! Vive la grande compagnie !" 30 Ce genre de trahison est beaucoup plus en accord avec le tempérament de l'intendant que les tractations imaginées par Marmette et Rousseau. Il colle assez à la réalité du personnage pour être tout à fait vraisemblable. De plus, il offre le grand avantage de dégager la responsabilité de l'Angleterre; les Anglais ne seraient pas seuls à avoir recherché la guerre.

Mais Bigot n'est pas le seul coupable; la France l'est tout autant que lui. Elle n'a d'abord pas su apprécier le courage et le dévouement des Canadiens. Elle a exigé d'eux plus qu'ils ne pouvaient donner: "Ils ne peuvent pas, après tout, affirme La Galissonière, faire l'impossible, et c'est pourtant ce qu'exige la France. Elle veut qu'ils livrent ses batailles, labourent ses champs, puis donnent, pour obéir aux ordonnances nouvelles de l'intendant, le pain de leur modeste table ${ }^{31}$." Toujours confiant en la vieille devise: "Si les cieux s'écroulaient sur nos têtes nous devons, en vrais Gaulois, les retenir sur la pointe de nos lances", les coloniaux ont tenté l'impossible pour la mère patrie. Mais cette dernière, en marâtre qu'elle est, n'a pas su l'apprécier; elle est restée sourde à leurs appels. Les Canadiens en sont profondément meurtris dans leurs sentiments patriotiques: "Ils se sentaient moitié délaissés et tout à fait dédaignés par la mère patrie ${ }^{32}$."

Lors de la révolution américaine, la France a de nouveau montré qu'elle ne tenait pas aux Canadiens. Si elle avait réellement voulu reprendre la Nouvelle-France, la révolte des colonies américaines lui offrait une occasion facile. Que fit-elle alors ?

${ }^{29}$ W. Kirby, Le Chien d'Or (2 vol., Québec, 1926), I : 275.

30 Ibid., I: 275.

31 Ibid., I: 29.

32 Ibid., II : 179. 
Elle se porta au secours d'anciens ennemis, mais n'eut pas la moindre velléité de délivrer ses enfants :

Comment cette France si cruellement sourde à leurs supplications, cette France si vilement indifférente à leurs souffrances et à leur héroïsme, accourait à la voix des étrangers !...Ah ! l'honneur se révoltait, l'âme s'indignait et le soldat canadien ne pouvait pas marcher sous les mêmes drapeaux que ceux qui avaient été ses ennemis constants ou ses maîtres oublieux ${ }^{33}$.

La France se montrait dénaturée; l'influence des philosophes achevait de la pourrir ${ }^{34}$.

Toutes les hontes de la défaite doivent donc retomber sur Bigot et sur la France sans éclabousser pour autant le nom des Canadiens. D'ailleurs les Anglais le reconnaissent eux-mêmes: "Ce ne sont pas les armées anglaises qui ont pris Québec et forcé Montréal à capituler, c'est la rapacité, c'est le brigandage de Bigot! C'est la coupable indifférence de la luxurieuse cour de Versailles ${ }^{35}$."

William Kirby utilise une argumentation à double tranchant. D'un côté, il réhabilite les Canadiens français, mais de l'autre, il vilipende la France. Reporter sur la mère patrie et l'administration coloniale toute la responsabilité de la défaite sauve l'honneur des Canadiens mais contribue à rompre les liens affectifs qui subsistent encore entre les anciens sujets et la mère patrie. D'une pierre deux coups: les Canadiens reprennent confiance en euxmêmes et les Anglais gagnent leur sympathie.

La manœuvre de Kirby se révèle particulièrement au loyalisme qu'il prête aux Canadiens français. Parce qu'ils ont compris le triste sort que la France leur avait fait, les vaincus de 1760 se sont tournés vers l'Angleterre: "Le peuple de la NouvelleFrance savait qu'il avait été pillé, volé, ruiné par l'intendant Bigot, puis lâchement abandonné par son roi; alors il s'était

33 Ibid., II : 287.

34 Ibid., II : 162.

35 Ibid., II : 385. 
tourné avec espoir vers le vainqueur et l'avait accepté franchement, comme l'arbitre de ses destinées nouvelles ${ }^{36}$." Par la suite, le loyalisme des Canadiens ne s'est jamais démenti. Jouant le prophète, le voyageur suédois Kalm augure ainsi de la fidélité des Canadiens français:

Je vois venir un jour, repartit Kalm, où les colonies anglaises se révolteront et secoueront le joug de l'Angleterre ! Je vois venir un jour où les colonies anglaises voudront proclamer leur indépendance. Alors, elles tendront vers vous des mains suppliantes, car elles auront besoin d'amis et de secours !... Et la Nouvelle-France devenue province anglaise ne les écoutera pas et détournera la tête ! Elles vous demanderont le secours de votre épée, de la Corne de SaintLuc, le secours de votre épée, Rigaud de Vaudreuil ! et vous les repousserez ! Vous resterez fidèles à votre nouveau souverain !... et vienne un temps où l'Angleterre, lâche et dégénérée, vous abandonnera comme le fera bientôt la France, le dernier coup de canon qui sera tiré pour la défense de son drapeau, le sera par un Canadien français ${ }^{37}$.

Les anciens sujets de la France n'ont rien perdu au change. La protection de l'Angleterre leur offre un brillant avenir. Avec leurs compatriotes de langue anglaise, ils pourront former une race supérieure en unissant les cultures des deux peuples les plus civilisés du monde: "Il faudrait, dit Kalm, pour former une civilisation digne de ce grand continent, que la courtoisie et l'urbanité du peuple français pussent s'unir à la rude énergie de l'Anglais. Heureux le pays où les qualités de ces deux grands peuples se fondront ensemble ! et je crois l'entrevoir ce pays dans les ombres de l'avenir ! . . . ${ }^{38}$ "

Les intentions de Kirby ne font point de doute. De tous les romanciers canadiens-anglais, il est le seul à pénétrer aussi profondément la mentalité canadienne-française. Un point de notre psychologie a retenu son attention: notre complexe d'infériorité. Pas d'unité canadienne possible, selon lui, avant la guérison de

36 Ibid., II : 386.

37 Ibid., II : 172.

38 Ibid., II : 171. 
ce traumatisme. C'est pourquoi il s'emploie à rendre à ses compatriotes de langue française une meilleure estime d'eux-mêmes en incriminant Bigot et la France. Mais ce n'est là qu'un aspect du but poursuivi. Les Canadiens français n'accompliront leur destinée qu'en s'associant aux Canadiens anglais pour former une seule grande nation. Au fond, n'est-ce pas un peu la pensée de Durham rajeunie et mieux présentée ?

De 1925 à 1927, Jean Féron publie une série de cinq romans sur la guerre de conquête sous le titre général de Flambard. Il y charge Bigot de tous les crimes et de toutes les trahisons. Comme Kirby, il imagine deux camps qui se livrent une lutte à mort. D'un côté, Bigot, Péan, Varin, Colpron, Estèbe, Pénissault, Descheneaux et tous les profiteurs de guerre; de l'autre, le capitaine Vaucourt, Flambard et tous les loyaux sujets de Sa Majesté. Le groupe des concussionnaires jouit d'une puissance presque illimitée. Comme intendant, Bigot régente le commerce, la police, la justice et les douanes. Pour endormir la vigilance du trop pusillanime marquis de Vaudreuil, il a soin de l'entourer de personnes entièrement dévouées à ses intérêts. Il est même prémuni contre les dénonciations en haut lieu; il peut opérer impunément sous la protection de la toute-puissante Pompadour. En face de cette clique formidable, un petit capitaine de milice et un grenadier français.

Poursuivant en cela la tradition, Féron affirme que Bigot et sa bande voient dans la guerre de Sept ans une occasion unique de s'amasser une fortune colossale. Mais Vaucourt et Flambard sont là pour dénoncer leurs manigances. Aussi les concussionnaires vont-ils tout mettre en œuvre pour faire disparaître les patriotes ou du moins les réduire à néant. Ils vont d'abord tenter d'assassiner Vaucourt, mais sans succès. Ensuite, ils vont enlever l'épouse et le fils de Vaucourt. Les deux factions se combattent avec des alternances de succès et de revers pendant cinq tomes. Les péripéties sont telles qu'on est tenté de dire avec Flambard: "Eh bien ! je suis fatigué de vos trappes et de vos caves, de vos fournaises et de vos citernes... (39)". Sans les échéances que

39 J. Féron, Le Siège de Québec (Montréal, E. Garand, 1927, vol. XXXII, coll. "Roman canadien"), 70. 
dressent les événements, ces combats pourraient s'éterniser en un immense roman-fleuve. Mais les Anglais sont là qui assiègent Québec.

En effet le siège s'éternise et les Anglais vont manquer de vivres. Un émissaire rencontre en secret les officiers de la Grande Compagnie et pendant que les troupes sont réduites à la portion congrue et que le peuple meurt de faim, Bigot et sa bande ouvrent aux ennemis les magasins du roi. Mais Flambard découvre la cache des vivres avant que les Anglais n'en prennent possession. Il révèle la trahison au gouverneur. La bande est toutefois si puissante qu'elle parvient à circonvenir Vaudreuil.

Les troupes anglaises ont eu beau détruire presque complètement la ville de Québec, ravager toutes les campagnes du bas $\mathrm{du}$ fleuve, elles n'ont pas réussi à provoquer la défection des Canadiens. Wolfe, quasi découragé, voit venir l'automne avec appréhension. Il ne pourra emporter la ville sans le concours des traîtres. Il entre de nouveau en contact avec Bigot et s'assure de la complicité de Vergor. Flambard a beau avertir Montcalm du danger que court la ville du côté du Foulon, personne ne veut le croire. Et c'est la défaite des Plaines d'Abraham. Mais la trahison de Bigot ne s'arrête pas à l'affaire du Foulon.

La victoire des Plaines n'octroyait pas pour autant la ville de Québec aux ennemis. Retranchée derrière les remparts, "la garnison pouvait tenir encore une dizaine de jours et attendre l'arrivée de Lévis à la tête de l'armée entièrement reformée" (40). Tout espoir n'était donc pas perdu. Bigot risquait de voir ses plans déjoués; il imagina le stratagème suivant. Il ferait remettre à $M$. de Ramesay un ordre faussement signé de Lévis lui enjoignant de livrer la ville au plus tôt. M. et Mme Péan se rendraient à Trois-Rivières et feraient croire, à leur retour, qu'ils tiennent l'ordre du nouveau général. Un seul obstacle se dressait en face des conjurés: Flambard chevauchait vers Montréal pour avertir Lévis de la mort de Montcalm et de ses responsabilités nouvelles. Il fallait done le rattraper au plus tôt. Mais

40 J. Féron, Le Drapeau blanc (Montréal, E. Garand, 1927, vol. XXV, coll. "Roman canadien"), 38. 
Flambard parvient à déjouer toutes les ruses de l'ennemi sauf la dernière. Péan et sa femme parviennent à Québec avant lui et quand il arrive avec l'ordre de Lévis de résister jusqu'à la venue des troupes, Ramesay a déjà rendu la ville aux Anglais.

Féron a l'avantage de venir après les autres; il connaît les interprétations traditionnelles et ne craint pas de leur donner une forme des plus affirmatives. Selon lui, Bigot n'avait qu'une intention, trahir. Depuis le début des hostilités, il s'y efforçait mais sans succès. A la longue, à force d'affaiblir la colonie par tous les moyens, il devait y réussir. Ainsi, pendant toute la guerre de Sept ans, l'effort de guerre des Canadiens a-t-il été sournoisement miné par les concussionnaires sans jamais toutefois démentir la réputation de courage et de bravoure des vaincus.

Les romans que nous avons groupés sous le titre trahison de Bigot ont ceci de commun: ils exaltent tous l'héroïsme des Canadiens pendant la guerre de la conquête, moins toutefois par le rappel des actions d'éclat que par la description des conditions difficiles qui ont entouré la résistance. Tous les romanciers incriminent copieusement Bigot et ses satellites et blâment la conduite de la France. Parmi eux, Marmette, Rousseau et Jean Féron accusent formellement Bigot de trahison. Adèle Bibeau et Mme Leprohon n'en excluent pas non plus la possibilité. William Kirby, pour sa part, reporte toute la responsabilité de la défaite sur la France.

Quelle conclusion tirer de ces constatations ? De l'analyse qui précède ressort une constante: tous les romanciers qui traitent de Bigot s'emploient à exonérer les Canadiens de tout blâme dans la défaite de 1760. D'instinct, ils sentent où blesse le bât. De la défaite originent toutes les humiliations, tous les mépris. Ils reconnaissent que c'est là l'événement qui a marqué le plus profondément la psychologie canadienne. L'échec des armes françaises et le succès des Britanniques a en quelque sorte convaincu les Canadiens de leur infériorité. La défaite a déterminé un complexe. Comment le guérir?

Vanter les Canadiens n'y ferait rien: une défaite reste toujours une défaite. Dans un cas pareil, la littérature recourt à 
un procédé que nos auteurs ignoraient peut-être mais qui confirme bien leur conception toute médiévale de l'honneur: le héros invincible par définition ne peut succomber que par trahison. C'est dans la Chanson de Roland que nous trouvons l'illustration la plus parfaite de ce procédé.

Charlemagne, l'empereur à la barbe fleurie, a courbé sous son sceptre toutes les nations de l'Europe. Seul l'émir Baligan lui résiste. Il passe les Pyrénées pour réduire le dernier des infidèles. Mais au retour son arrière-garde est anéantie au col de Ronceveaux. Cette défaite, somme toute insignifiante au cours d'un règne si fertile en victoires, est pourtant le seul fait d'armes qu'a retenu la légende pour le magnifier à l'égal des plus belles victoires. Toute la gloire dont s'était couvert le vieil empereur ne comptait plus puisqu'une défaite ternissait sa renommée. Charlemagne l' "Invictus" ne pouvait pas avoir été vaincu. Il avait donc été trahi. C'est alors que les bardes imaginèrent la trahison de Ganelon.

Depuis le début de ce travail, le lecteur a été à même de constater la façon dont les Canadiens voient leur passé: une succession de victoires, et pas n'importe lesquelles; les Canadiens ont toujours été vainqueurs dans les circonstances les plus défavorables, infériorité numérique, infériorité d'armement. Comme le dit Rousseau et le répète Kirby: un Canadien ne vaut-il pas dix Anglais? Comme les empereurs romains, comme les héros légendaires, le titre d' "invictus" semble-t-il, leur revient de droit. Comment concilier avec cette notion le statut de vaincu qui est celui des Canadiens depuis la conquête? Pas d'autres explications, les Canadiens ont été trahis.

A la simple lecture de l'histoire, cette explication vient spontanément à l'esprit de Marmette; Rousseau et Féron la corroborent. Adèle Bibeau, William Kirby insistent sur l'abandon de la colonie par la France. Le résultat est le même. La France a trahi le Canada. Dans des circonstances normales les Anglais n'auraient pu vaincre les Canadiens.

Centre des études universitaires

MAURICE LEMIRE Trois-Rivières, Qué. 